\title{
Abnormalities of Serum Lipid Profile in Type 2 Diabetes Mellitus in North-Western Nigeria
}

\author{
A.Yakubu, S. Dahiru, A.S. Mainasara, P.O. Anaja, B. Musa, H.A. Hassan
}

\begin{abstract}
Background:Dyslipidaemia has been indicted in type-2 diabetes. It increases the patient's risk of developing cardiovascular diseases. This study was conducted to compare the lipid profile of diabetic patients and healthy controls.

Method and Method: A case - control study was conducted. 80 type 2 diabeticpatients and 80 controls were recruited in the study. Serum lipid profiles: total cholesterol, triglycerides, low density lipoprotein, and high density lipoprotein, anthropometry,glycatedhaemoglobinand fasting blood glucose were assessed using standard methods. Epi Info Version 7 was used for statistical analysis and $P<0.05$ was considered to be statistically significant.

Results: Compared to the control subjects, diabetic patients had statistically significant higher levels of total cholesterol( $p=$ 0.001), triglycerides $(p=0.001)$, Low density lipoprotein $(p<0.05)$, high .density lipoprotein $(p=0.001)$, HbA1c $(P=)$ and the fasting blood glucose $(P=)$.Among the diabetic subjects, the dyslipidemia shows a gender dimorphism with diabetic females having higher levels compared to the diabetic males $(P=) .49 \%$ of the studied diabetics were obese. Obese diabetics had significantly higher serum levels of total cholesterol, low density lipoprotein and high density lipoprotein $(p=0.05)$ compared to non-obese diabetics. The duration of diabetes also affects the serum lipid profile in such a way that diabetics with greater than 5 years history of diabetes duration having/ had higher total cholesterol levels compared to those with $\leq 5$ years history of diabetes $(P=)$ or lower diabetes duration.

Conclusion: Type 2 diabetic patients have higher risk of having dyslipidemia compared to the control group. Female gender, obesity and greater than 5 year's history of diabetes higher diabetes duration were associated with dyslipidemia in diabetic patients. We recommend routine lipid profile test especially in the higher risk groups.
\end{abstract}

Index Terms - Type 2 Diabetes, Controls, Dyslipidemia.

\section{INTRODUCTION}

Diabetes Mellitus (DM), commonly referred to as diabetes, is a group of metabolic diseases in which there is high blood glucose levels over a prolonged period.[1]The hyperglycaemia is resulting from defects in insulin secretion, insulin action, or both.[2]

Dr. Abdulmumini Yakubu, Senior Lecturer Department of Internal Medicine, Faculty of Clinical Sciences, College of Health Sciences, Usmanu Danfodiyo University Sokoto, Nigeria

Dr. Shafiu. Dahiru,Nigeria Field Epidemiology and Laboratory Training Program (NFELTP), Asokoro Abuja - Nigeria,

Dr. AbdulllahiSulaiman. Mainasara,Department of Chemical Pathology, Faculty of Basic Clinical Sciences, College of Health Sciences, Usmanu Danfodiyo University, Sokoto,

Prof. PeterOcheni. Anaja,Department of Chemical Pathology, Faculty of Medicine, Ahmadu Bello University, Zaria-Nigeria,

Dr. Badamasi. Musa, State Primary Healthcare Development Agency (SPHCDA), Dutse - Jigawa State,

Dr. HaliruAbdullahi. Hassan, Department of Chemical Pathology, Faculty of Basic Health Sciences, Bayero University Kano
Long-term complications of diabetes are usually the result of problems with blood vessels; due to uncontrolled hyperglycemia that remains over a long period of time which causes both the small and large blood vessels to narrow, mainly as a result of complex sugar-based substances that build-up in the walls of blood vessels, leading to microvascular and macrovascular diseases.[3]

Dyslipidemia, a condition characterized by elevation of plasma total cholesterol, triglycerides (TGs), or both, or a low or high-density lipoprotein levels that contributes to the development of atherosclerosis [4], is associated with diabetes.Studies on the pathogenesis of type 2 DM have shown that hyperlipidaemia, which is frequently present in diabetes, can cause insulin resistance and $\beta$-cell dysfunction (lipotoxicity) [5]. Patients with Type 2 diabetes are frequently affected by atherosclerotic vascular disease. These patients often have abnormalities of both quantity and quality of lipoproteins, which, among other factors, might be responsible for the increased incidence of vascular complications [6]

Common abnormalities of lipids in diabetes were shown to includeshypertrigyceridaemia, with or without associated increase in plasma cholesterol (7), elevated LDL cholesterol.[8] and decreased HDL cholesterol.[9] There may be differences in the lipid profile pattern from patient to patient or from population to population as a result of difference in genetic composition among other factors.[10]The present study is therefore conducted to determine how lipid profile of diabetic patients is affected by the disease.

\section{MATERIALS AND METHODS}

\section{Study Design}

A 1:1 sex matched case-control study was conducted. Structured questionnaire was used as a data tool to obtain the socio-demographic and clinical information of the respondents. Biochemical tests were also conducted using standard methods.

\section{STUDY SUBJECTS}

Patients who visited the diabetic clinics of RasheedShakoni Specialist Hospital Dutse - Jigawa State, and who is diagnosed with type 2 diabetic mellitus were used as cases. Those diagnosed not have diabetes from the same hospital were used as controls.

\section{ANTHROPOMETRY MEASUREMENTS}

Height measurement was made using stadiometer (model 220, SecaGmbh and Co., Germany). Weight of the 
respondents was taken while wearing light clothing, to the nearest $0.1 \mathrm{~kg}$ by using weighing health scale (model ZT 120 , SecaGmbh and Co., Germany). The waist circumference was measured using a stretch-resistant tape; Hip circumference was measured with the tape parallel to the floor. For both measurements, subjects were made to stand with feet close together, arms at the side and weight evenly distributed. Blood pressure was measured using sphygmomanometer.

\section{BIOCHEMICAL MEASUREMENTS}

FBG was measured by the glucose oxidase method [11 12]; glycatedhaemoglobin by ion exchange resin method [13];serum total cholesterol, HDL cholesterol and triglycerides were measured by enzymatic colorimetric method [14 - 15], using commercially prepared kit from Randox Company; while serum LDL was calculated by Friedewald's Equation[16].

\section{ETHICAL CONSIDERATION}

The ethical clearance for the study was obtained from the Research and Ethics Committee of the RasheedShakoni Specialist Hospital, Dutse. Informed consent of the recruited subjects was obtained.

\section{Statistical ANALYSIS}

Data was cleaned and analyzed using Epi Info for windows version 7. Data are expressed as mean \pm standard error of the mean SEM. For all assessment a value of $\mathrm{p}<0.05$ was accepted as statistically significant.

\section{RESULT}

Eighty diabetic patients and eighty gender-matched controls were studied. (Table1) gives the anthropometry data of the study subjects. Mean Age \pm SEM of the diabetic patients was $49.14 \pm 1.62$ while that of controls was $46.16 \pm$ 1.70. Mean waist-to-hip ratio (WHR) of the diabetic patients was significantly higher than that of controls $(\mathrm{p}<0.05)$. Table 2 gives the mean lipid profile and glycaemic control indicators of the diabetic patients and controls. Diabetic patients when compared to controls showed statistically significant increase in serum levels of total cholesterol $(\mathrm{p}<0.001)$, LDL cholesterol $(\mathrm{p}<0.05)$, HDL cholesterol $(p<0.001)$ and triglycerides $(p<0.001)$. Of the eighty diabetic patients studied, 24 had five years or less diabetes duration while the remaining 56 had more than five years diabetes duration. Serum total cholesterol was significantly higher $(\mathrm{p}<0.05)$ in those with more than five years diabetes duration (Table 3).Using WHR as a measure of obesity, $49 \%$ of the studied diabetics were obese. Serum total cholesterol, LDL cholesterol, HDL cholesterol as well as triglycerides were significantly higher in obese diabetics compared to non-obese diabetics (table 4).

Table 1: Anthropometric Data of the Study Subjects (Mean \pm SEM)

\begin{tabular}{|c|c|c|c|}
\hline \multicolumn{1}{|c|}{ Variables } & Diabetics & Controls & $\mathrm{p}-$ Value \\
\hline $\mathrm{n}$ & 80 & 80 & 0.103 \\
\hline Age(years) & $49.14 \pm 1.62$ & $46.16 \pm 1.70$ & 0.398 \\
\hline BMI $\left(\mathrm{kg} / \mathrm{m}^{2}\right)$ & $25.69 \pm 0.57$ & $25.10 \pm 0.87$ & 0.024 \\
\hline WHR & $0.90 \pm 0.01$ & $0.87 \pm 0.01$ & 0.001 \\
\hline SBP $(\mathrm{mmHg})$ & $129.00 \pm 2.10$ & $120.63 \pm 1.37$ & 0.469 \\
\hline DBP $(\mathrm{mmHg})$ & $78.13 \pm 1.30$ & $76.88 \pm 1.13$ & \\
\hline
\end{tabular}

SEM = Standard Error of Mean; $\mathrm{n}=$ Number of Subjects; BMI= Body Mass Index; WHR= Waist to Hip Ratio; SBP = Systolic Blood Pressure; DBP = Diastolic Blood Pressure; $\mathrm{P}<0.05$ is Significantly Different.

Table 2: Lipid Profile and Blood Glycaemic Control Indicators of the Study Subjects (Mean \pm SEM)

\begin{tabular}{|c|c|c|c|}
\hline Variables & Diabetics & Controls & p - Value \\
\hline FBG(mmol/L) & $9.32 \pm 0.54$ & $4.18 \pm 0.08$ & 0.000 \\
\hline HbA1c(\%) & $10.69 \pm 0.25$ & $5.37 \pm 0.12$ & 0.000 \\
\hline T. Chol(mmol/L) & $4.76 \pm 0.13$ & $3.95 \pm 0.13$ & 0.000 \\
\hline HDL Chol(mmol/L) & $0.84 \pm 0.04$ & $0.62 \pm 0.03$ & 0.025 \\
\hline LDL Chol(mmol/L) & $3.04 \pm 0.11$ & $2.67 \pm 0.12$ & \\
\hline
\end{tabular}




\begin{tabular}{|l|c|c|c|}
\hline Triglycerides $(\mathrm{mmol} / \mathrm{L})$ & $1.92 \pm 0.09$ & $1.43 \pm 0.06$ & 0.000 \\
\hline AI & $0.3592 \pm 0.031$ & $0.3958 \pm 0.029$ & 0.395 \\
\hline
\end{tabular}

$\mathrm{n}$ = Number of Subjects; $\mathrm{T}$. Chol $=$ Total Cholesterol; TGs = Triglycerides; HDL= High Density Lipoprotein; LDL= Low Density lipoprotein; $\mathrm{AI}=$ Atherogenic Index $\left(\mathrm{AIP}=\log _{10} \mathrm{TGs} / \mathrm{HDL}\right) ; \mathrm{SEM}=$ Standard Error of Mean. $\mathrm{P}<0.05$ is Significantly Different.

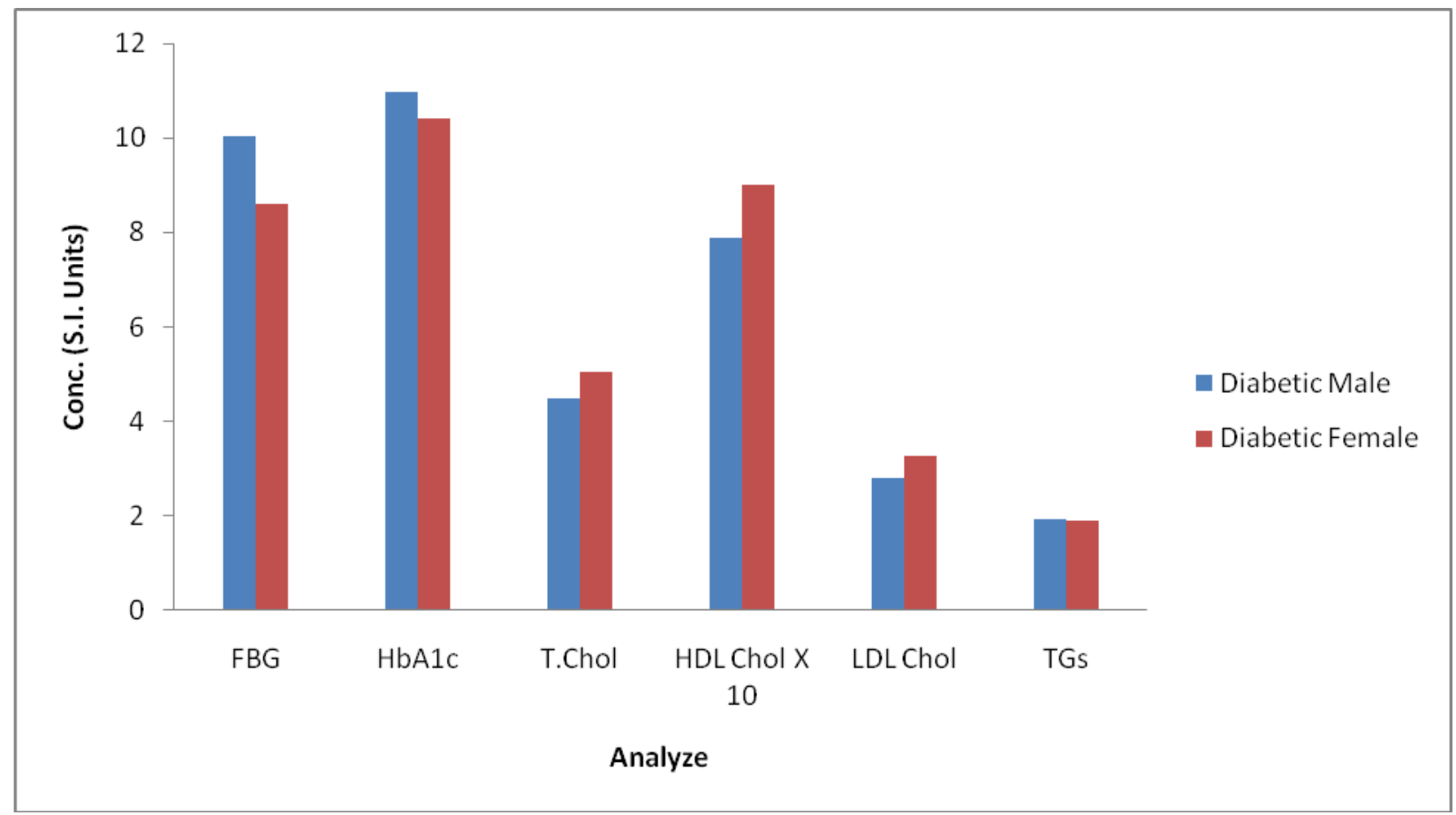

Figure 1: Gender Based Biochemical Parameters of the Diabetic Subjects

Table 3: Effects of Duration of Diabetes on Biochemical analyze of Diabetic Patients (Mean \pm SEM)

\begin{tabular}{|c|c|c|c|}
\hline \multicolumn{1}{|c|}{ Variables } & Diabetics & Diabetics & p - Value \\
& $\leq 5$ Years & 56 & 0.849 \\
\hline $\mathrm{n}$ & 24 & $9.38 \pm 0.65$ & 0.712 \\
\hline FBG(mmol/L) & $9.16 \pm 0.98$ & $10.76 \pm 0.30$ & 0.015 \\
\hline HbAlc(\%) & $10.55 \pm 0.47$ & $4.97 \pm 0.15$ & 0.095 \\
\hline T. Chol(mmol/L) & $4.28 \pm 0.22$ & $0.88 \pm 0.04$ & 0.091 \\
\hline HDL Chol(mmol/L) & $0.75 \pm 0.07$ & $3.16 \pm 0.13$ & 0.099 \\
\hline LDL Chol(mmol/L) & $2.75 \pm 0.20$ & $2.02 \pm 0.10$ & \\
\hline Triglycerides(mmol/L) & $1.68 \pm 0.19$ & & \\
\hline
\end{tabular}

$\mathrm{n}$ = Number of Subjects; FBG = Fasting Blood Glucose; HbA1c = GlycatedHaemoglobin; $\mathrm{T}$. Chol = Total Cholesterol; TGs = Triglycerides; HDL= High Density Lipoprotein; LDL= Low Density Lipoprotein; SEM = Standard Error of Mean. $\mathrm{P}<0.05$ is Significantly Different. 
Abnormalities of Serum Lipid Profile in Type 2 Diabetes Mellitus in North-Western Nigeria

Table 4: Effects of Obesity on Biochemical analyses of Diabetic Patients (Mean \pm SEM)

\begin{tabular}{|c|c|c|c|}
\hline \multirow{2}{*}{ Variables } & Obese & Non-obese & $\mathrm{p}-$ Value \\
& Diabetics & Diabetics & \\
\hline $\mathrm{n}$ & 39 & 41 & 0.648 \\
\hline FBG(mmol/L) & $9.06 \pm 0.67$ & $9.56 \pm 0.84$ & 0.880 \\
\hline HbA1c(\%) & $10.73 \pm 0.35$ & $10.67 \pm 0.37$ & 0.001 \\
\hline T. Chol(mmol/L) & $5.21 \pm 0.21$ & $4.33 \pm 0.13$ & 0.001 \\
\hline HDL Chol(mmol/L) & $0.96 \pm 0.05$ & $0.73 \pm 0.04$ & 0.007 \\
\hline LDL Chol(mmol/L) & $3.33 \pm 0.18$ & $2.75 \pm 0.11$ & 0.462 \\
\hline Triglycerides(mmol/L) & $1.99 \pm 0.13$ & $1.85 \pm 0.13$ & \\
\hline
\end{tabular}

$\mathrm{n}=$ Number of Subjects; SEM = Standard Error of Mean; FBG = Fasting Blood Glucose; HbA1c = GlycatedHaemoglobin; $\mathrm{T}$. Chol $=$ Total Cholesterol; TGs $=$ Triglycerides; $\mathrm{HDL}=$ High Density Lipoprotein $; \mathrm{LDL}=$ Low Density Lipoprotein. $\mathrm{P}<0.05$ is Significantly Different.

\section{Discussion}

Dyslipidaemias are frequently seen in type-2 diabetics. It makes an individual more prone to atherosclerosis and CHD. In the present study, all the lipid fractions are abnormally elevated in diabetics when compared with controls. This is in agreement of findings made in various studies $[17 ; 9 ; 6]$. Lipid metabolism and carbohydrate metabolism are highly connected in the human body. In diabetes, there is much derangement in carbohydrate metabolism which subsequently affects lipids metabolism and blood lipid levels. For instance, Insulin resistance is a primary defect in the majority of patients with T2DM and several studies showed that insulin affects the liver apolipoprotein production and regulates the enzymatic activity of lipoprotein lipase and cholesterol ester transport protein causing dyslipidemia [17].

In this study also, we observe gender dimorphism in the serum lipid profile of the diabetic patients. In non-diabetic condition, Wang et al.[2011]suggested that females have greater high-density lipoprotein (HDL) cholesterol concentration and lower low-density lipoprotein (LDL) cholesterol, very low-density lipoprotein (VLDL) cholesterol, total plasma triglyceride, and VLDL triglyceride concentrations (both during fasted and fed conditions) than age-matched males [18]. In our study,however,diabetic females had a higher total cholesterol, LDL cholesterol and HDL cholesterol compared to the diabetic males. This is in agreement with the findings of Gustafsson et al. in 2004 [19] and supported the findings made by Lee et al. 2000 [20], which shows that the impact of diabetes on the risk of coronary death is significantly greater for women than men.

Glycaemic control and the duration of diabetes were also associated with dyslipidemia in diabetes. In the current study, diabetics with more than five years diabetes duration have significantly higher total cholesterol level when compared to those with five years and below diabetes duration. Differences in the concentrations of the other fractions were not significant.We also compare obese diabetics with non-obese diabetics. Higher total cholesterol, LDL cholesterol and HDL cholesterol was found in obese diabetics than the non-obese. This could be justified by the fact that increased in whole-body adiposity and central fat accumulation and decreased insulin sensitivity are associated with increased plasma triglyceride and LDL cholesterol and decreased HDL cholesterol concentrations [21].

\section{REFERENCES}

[1] WHO (2014). "About Diabetes". World Health Organization. Retrieved 4 September, 2014.

[2] American Diabetes Association (2010). Diagnosis and Classification of Diabetes Mellitus. DiabCare.; 33 (1).

[3] Michael L.B., Edward P.F. and Larry E.S. (2010). Clinical Chemistry: Techniques, Principles, Correlations. Sixth Edition. Lippincott Williams \& Wilkins - London.

[4] Goldberg Anne Carol (2015). Dyslipidemia (Hyperlipidemia). Merck Sharp \& Dohme Corp http://www.merckmanuals.com/professional/endocrine-and-metabolic -disorders/lipid-disorders/dyslipidemia

[5] Saltiel, A.R., Kahn, C.R. (2001) Insulin signalling and the regulation of glucose and lipid metabolism. Nature 414:799-806.

[6] Zargar, A.H. et al., 1995. Serum Lipid Profile in Non-insulin-dependent Diabetes Mellitus Associated with Obesity. , 15, pp.9-13.

[7] Goldberg R.B. (1981). Lipid disorders in diabetes. DiabCare.; 4 : 561.

[8] Pradesh, A., 2012. Lipid Profile Levels in Type 2 Diabetes Melli- tus from the Tribal Population of Adilabad in. , pp.0-3.

[9] Uttra, K.M. et al., 2011. Lipid Profile of Patients with Diabetes mellitus ( A Multidisciplinary Study ). , 12(9), pp.1382-1384.

[10] Deurenberg P, Yap M, van Staveren WA. Body mass index and percent body fat: a meta analysis among different ethnic groups. Int J ObesRelatMetabDisord 1998; 22:1164-71.

[11] Trinder P. (1969). Determination of glucose in blood using glucose oxidase with an alternative oxygen receptor. Ann. Clin. Biochem. 6, 24 $-27$.

[12] Bauminger B.B. (1974). Micromethod for manual analysis of true glucose in plasma without deproteinization. J. ClinPathol.; 27 (12); 1015 - 1017.

[13] Trivelli L.A., Ranny H.M., Lai H.T. (1971). Glycosylated Haemoglobin. New Eng. J. Med.; 284:353.

[14] Allain C.C., Poon L.S., Chan C.S.G., Richmond W. and Fu P.C. (1974). Total Cholesterol. Clin. Chem.; 20: 470.

[15] Rieschlau, P., Bernt, E. and Gruber, W. (1974). Z KlinChemKlinBiochem, 12: 403. 
[16] Friedewald W.T., Levy R.I., Fredrickson D.S. (1972). Estimation of the concentration of low- density lipoprotein cholesterol in plasma, without use of the preparative ultracentrifuge. Clin. Chem.; 18: 499-502.

[17] Ozder, A., 2014. Lipid profile abnormalities seen in T2DM patients in primary healthcare in Turkey : a cross-sectional study. Lipids in Health and Disease, 13(1), pp.1-6.

[18] Wang, X., Magkos, F. \&Mittendorfer, B., 2011. Sex differences in lipid and lipoprotein metabolism: it's not just about sex hormones. J ClinEndocrinolMetab, 96(4), pp.885-893.

[19] Gustafsson I, Brendorp B, Seibaek M, Burchardt H, Hildebrandt P. The influence of diabetes and the diabetes - gender interaction on the risk of death in patients who were hospitalized with congestic heart failure. J.AM.Coll.Cardiol 2004; 43(5):771-77.

[20] Lee, W.L. et al., 2000. Impact of diabetes on coronary artery disease in women and men: a meta-analysis of prospective studies. Diabetes care, 23(7) pp.962-8. Available http://www.ncbi.nlm.nih.gov/pubmed/10895847.

[21] Ferrannini E, Balkau B, Coppack SW, Dekker JM, Mari A, Nolan J, Walker M, Natali A, Beck-Nielsen H. 2007. Insulin resistance, insulin response, and obesity as indicators of metabolic risk. J Clin Endocrinol Metab 92:2885-2892 